\title{
Extracellular protein degradation on demand
}

A protein degradation platform enables the targeted depletion of extracellular and membrane-bound proteins.

\author{
$\mathrm{C}$ \\ ontrolling the abundance of a protein \\ of interest can be crucial to discovering \\ its function in a cell, modulating \\ specific cellular pathways, and even \\ treating disease. A number of approaches \\ have been developed that can rapidly, \\ efficiently and sometimes reversibly reduce \\ the abundance of a protein using various \\ strategies for sequestration, downregulation \\ or degradation. The strategies that involve \\ degradation, however, have largely been \\ limited to cytoplasmic proteins. \\ Carolyn Bertozzi and her research group \\ at Stanford University developed a strategy \\ for specifically degrading extracellular and \\ membrane-bound proteins of interest. Their \\ approach fills a gap in the methodological \\ toolbox for controlled protein degradation, \\ as these proteins represent roughly $40 \%$ of \\ protein-encoding genes.
}

Their method for targeting these types of proteins involves lysosome-targeting chimeras they have named LYTACs. LYTACs are made up of a protein-targeting moiety (small molecule or antibody) fused to glycopeptide ligands that bind and activate the cation-independent mannose6-phosphate receptor (CI-M6PR), a membrane protein that transports certain glycosylated proteins to lysosomes. Thus, LYTACs bridge a target protein with CI-M6PR, ultimately resulting in degradation of the target protein in the lysosome.

In proof-of-principle experiments, the researchers showed that LYTACs could improve uptake of NeutrAvidin-647, a fluorescently labeled, degradation-resistant protein, 5- to 6-fold over controls. Microscopy revealed that the targeted proteins colocalized with labeled lysosomes, as expected for proteins internalized via CI-M6PR. The researchers further demonstrated general applicability on a range of proteins, including epidermal growth factor receptor, CD71, and programmed death ligand 1 (PD-L1). As an added demonstration of LYTACs, the team used their method in a CRISPR interference screen, which revealed that the exocyst complex is essential for CI-M6PR-mediated cargo internalization.

\section{Rita Strack}

Published online: 1 September 2020 https://doi.org/10.1038/s41592-020-0952-3

Research paper

Banik, S. M. Lysosome-targeting chimaeras for degradation of extracellular proteins. Nature https://doi.org/10.1038/s41586-020-2545-9 (2020).

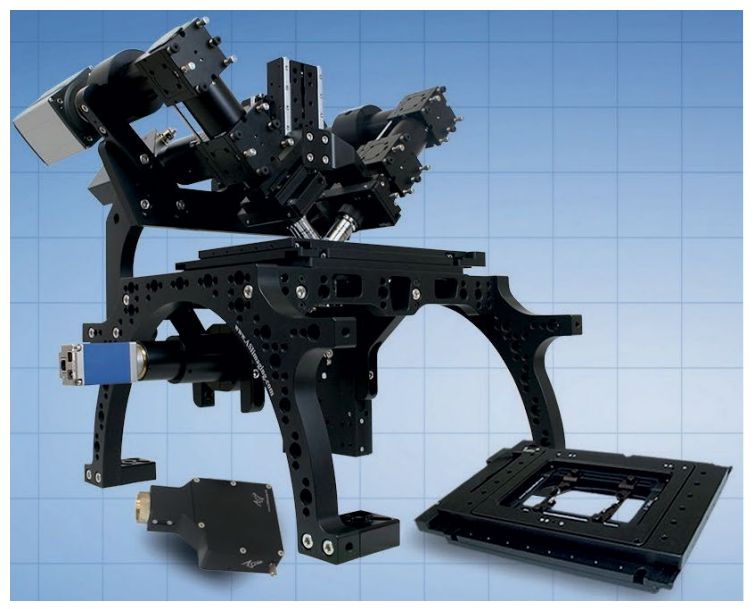

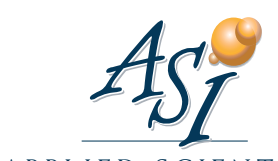

APPLIED SCIENTIFIC INSTRUMENTATION

www.asiimaging.com info@asiimaging.com

(800) 706-2284

(541) 461-8181

\section{We Create Solutions}

\author{
Ultra Precise Motion Control - D.C. Servo \\ motors down to $20 \mathrm{~nm}$, piezos down \\ to $1 \mathrm{~nm}$, and low drift XYZ stages.
}

Microscopy - Automation, modular microscopes, autofocus complete light sheet systems, and components.

\author{
OEM - Custom designed systems \\ to user specifications.
}

